\title{
The acute muscular effects of cycling with and without different degrees of blood flow restriction
}

\author{
D Kim ${ }^{1}$, JP Loenneke ${ }^{2}$, RS Thiebaud ${ }^{3}$, T Abe ${ }^{2}$, MG Bemben ${ }^{1}$ \\ ${ }^{1}$ Department of Health and Exercise Science, University of Oklahoma, Norman, USA \\ ${ }^{2}$ Department of Health, Exercise Science, and Recreation Management, Kevser Ermin Applied Physiology \\ Laboratory, University of Mississippi, University, USA \\ ${ }^{3}$ Department of Kinesiology, Texas Wesleyan University, Fort Worth, USA
}

Received: July 25, 2014

Accepted: July 31, 2015

\begin{abstract}
The aim was to compare the acute effects of work matched high intensity ( $75 \%$ peak aerobic capacity) aerobic exercise to low intensity ( $40 \%$ peak aerobic capacity) aerobic exercise with different degrees of blood flow restriction (BFR) [40\% estimated arterial occlusion (40 BFR) and 60\% estimated arterial occlusion (60 BFR)] on variables previously hypothesized to be important for muscle adaptation. There were no meaningful changes in torque. Anterior thigh muscle thickness was increased from baseline with high intensity cycling and 40 BFR $(\sim 2 \mathrm{~mm}$ increase, $p \leq 0.008)$. A significant increase in lactate occurred in all exercise conditions but was greatest with high intensity cycling $(\sim 5.4 \mathrm{mmol} / \mathrm{L}$ increase $)$. Muscle activation was significantly higher with high intensity cycling compared to low intensity cycling with BFR, regardless of pressure ( $\sim 25 \%$ vs. $\sim 12 \%$ MVC). Mean power frequency was not different between conditions but did increase from the first 5 minutes of exercise to the last 5 minutes $(93 \%$ vs. $101 \%, p<0.001$ ). Ratings of perceived exertion (RPE) were higher with high intensity cycling but discomfort was similar between conditions. We wish to suggest that high intensity cycling produces greater muscular stress than that observed with work matched low intensity cycling in combination with BFR.
\end{abstract}

Keywords: amplitude, swelling, KAATSU, muscle strength, muscle hypertrophy, hypoxia

It has been traditionally thought that one needs to consistently lift weights at approximately $70 \%$ of their one repetition maximum (1RM) to have noticeable increases in muscle size and strength (6). Although numerous positive effects have been observed from heavy resistance training, some populations (e.g. elderly, rehabilitating patients, etc.) might be advised not to perform high-load resistance training and may be limited to performance of low-load resistance exercise. However, this recommendation has been challenged over the past decade by evidence which suggests substantial increases in muscle mass and strength following low load resistance exercise taken to failure $(9,29,36)$ and submaximal low load resistance exercise completed in combination with blood flow restriction (BFR) (27).

Interestingly, aerobic exercise in combination with BFR has also been shown to produce significant increases in muscle size and strength although much less is known about this modality of exercise $(2,3,5)$. The reported mechanisms behind the muscular effects of aerobic exercise in combination with BFR are not completely known, but several hypotheses exist. A foundational mechanism may be the acute increase in muscle cell swelling $(17,31)$,

Corresponding author: Jeremy Paul Loenneke, $\mathrm{PhD}$

Department of Health, Exercise Science, and Recreation Management, Kevser Ermin Applied Physiology Laboratory, University of Mississippi

P.O. Box 1848, University, MS 38677, USA

Phone: 1-(662) 915-5567; Fax: 1-(662) 915-5525; E-mail: jploenne@olemiss.edu 
which is defined as an increase in muscle size coupled with the loss of plasma volume, suggesting a fluid shift from the vascular space into exercising muscles. To illustrate, acute muscle swelling has been observed with slow $(56 \mathrm{~m} / \mathrm{min})$ and fast BFR walking $(87 \mathrm{~m} / \mathrm{min})$ without a significant decrease in torque (31), suggesting that muscle fatigue may not be a prominent mechanism with low intensity aerobic exercise. Further, walking at slow speeds does not result in an accumulation of metabolites in the blood $(75 \mathrm{~m} / \mathrm{min})(24)$, although cycling at $40 \%$ of $\mathrm{VO}_{\max }$ does increase lactate levels over baseline values $(\sim 2 \mathrm{mmol} / \mathrm{L}$ increase) (13), albeit to a lesser extent than that observed with resistance training (26). Therefore, it appears that the predominant mechanism behind the benefits observed with BFR aerobic exercise at low intensities may be the acute increase in muscle cell volume and this increase in cell volume may also be responsible for the increased ERK $1 / 2$ and p38 phosphorylation and for the decreased phosphorylation of eEF2 observed in young men walking under BFR at $55 \% \mathrm{VO} 2_{\max }(32)$.

The studies showing positive effects of low intensity aerobic exercise are only being compared to low intensity exercise without BFR. Comparing the acute responses observed with low intensity aerobic exercise combined with BFR to those observed with high intensity aerobic exercise without BFR are important as both protocols have been shown to result in favorable skeletal muscle adaptations (12). Further, understanding acute differences between protocols may help with designing optimal studies in the future and/or provide information as to why the muscle response to low intensity aerobic exercise with BFR is less than that observed with low load resistance training in combination with BFR (27). Thus, the purpose of this study was to compare the effects of work matched high intensity aerobic exercise to low intensity aerobic exercise with different degrees of blood flow restriction on torque, muscle activation, mean power frequency (MPF), lactate, and muscle swelling; as these variables have been hypothesized as potential mechanisms behind the beneficial effects of this stimulus. In addition, the perceptual responses to each condition were investigated.

\section{Materials and Methods}

\section{Participants}

Ten physically active men aged 18-35 years were recruited to participate. Physically active was defined as being active three or more days per week with a whole body resistance training component two or more days per week for at least the last three months. Physically active participants were used to better reflect the actual acute responses to different exercises. The use of this population decreases the chance of eliciting a "training response" due to repeated testing which may occur if an untrained cohort was used. Participants who were hypertensive ( $>140 / 90 \mathrm{mmHg}$ ), used tobacco regularly within the past 6 months, or those who had more than one risk factor for thromboembolisms (30) were excluded from participating. Of those initial 10, only 9 completed all of the testing sessions. One participant had a change in his daily schedule that no longer allowed him to participate in the study. Thus, he was excluded from all further analyses. The study received approval from the institutional review board of the University of Oklahoma, and each participant gave written informed consent before participation.

\section{Study design}

During the initial screening visit, participants had their height (to the nearest $0.5 \mathrm{~cm}$ ) and body mass (to the nearest $0.1 \mathrm{~kg}$ ) measured to calculate body mass index (BMI). Due to potential safety concerns, if a participant had a BMI equal to or greater than $30 \mathrm{~kg} / \mathrm{m}^{2}$ along 
with another risk factor for thromboembolism, that participant was excluded (30). Next, blood pressure and ankle brachial index were measured in the supine position to exclude those who may be hypertensive or those who had indications of peripheral vascular disease. Following this, thigh circumference was measured in the supine position on the non-dominant leg to determine the pressure that would be used during the resistance exercise bouts with BFR. Participants were then familiarized with the BFR stimulus by having the cuffs inflated to $60 \%$ of their estimated arterial occlusion pressure (based on thigh circumference). Additionally, participants were familiarized with performing maximal voluntary contractions. Participants were then scheduled for Visits 2 and 3 which tested their peak aerobic capacity. Peak aerobic capacity was determined in order to set the workload for the remaining visits. Following the third visit participants were scheduled for their first of four (Visits 4-7) experimental visits (three exercise conditions, one control, Table I). Each visit was separated by approximately 5-10 days. To set condition order, a sequence generator (Random.org) was used where the smallest value was one and the largest value was four (corresponding to a condition in Table I). This was generated for each participant. The individual conditions will be abbreviated in the results and discussion as follows:

High: High Intensity cycling

40BFR: Low intensity cycling at $40 \%$ estimated arterial occlusion pressure.

60BFR: Low intensity cycling at $60 \%$ estimated arterial occlusion pressure.

CON: Non-exercise control.

Table I. Exercise protocols

\begin{tabular}{|l|c|c|c|}
\hline & \% VO2peak & \% Arterial occ. & Volume \\
\hline High & 75 & 0 & $2874(761)$ \\
\hline 40BFR & 40 & 40 & $2819(925)$ \\
\hline 60BFR & 40 & 60 & $2916(790)$ \\
\hline CON & 0 & 0 & 0 \\
\hline
\end{tabular}

$\%$ VO2peak $=$ percentage peak oxygen consumption; $\%$ Arterial Occ. $=$ percentage of estimated arterial occlusion; High = high intensity cycling; $40 \mathrm{BFR}=$ cycling under $40 \%$ estimated arterial occlusion; $60 \mathrm{BFR}=$ cycling under $60 \%$ estimated arterial occlusion pressure; $\mathrm{CON}=$ control

\section{Cycle exercise protocols}

Participants completed 4 protocols in random order (3 exercises and 1 non-exercise control). The protocols were comparing exercise intensity and different degrees of BFR on the acute muscular response. All participants but one maintained 50-60 RPM's for each exercise visit. One participant preferred a faster cadence ( 90 RPM) which he was able to maintain for the HIGH but he was unable to fully complete the 40BFR exercise session at this cadence (further discussed below). The differing degrees of BFR (40BFR and 60BFR) were chosen based on pressures used previously in our laboratory with low load resistance training (21). The protocols within each experiment are found in Table I. During the control conditions, participants rested quietly in a chair but did not exercise. Prior to each condition muscle thickness, whole blood lactate (WBL), hematocrit, and torque were measured in that order. In addition, immediately following each exercise bout torque, WBL, hematocrit and muscle thickness were measured again in that order. Muscle activation and MPF were measured at 
pre- and throughout exercise. Participants wore a polar heart rate belt (Polar T31 Coded ${ }^{\mathrm{TM}}$ Transmitter, Polar Electro, Sacramento, CA, USA) across the chest to monitor their heart rate (HR) throughout each session. The pre-measurements were made prior to putting the BFR cuffs on and the post-measurements were taken following cuff removal.

\section{Maximal voluntary isometric contraction}

The maximal voluntary isometric contraction (MVC) of the dominant knee extensor was performed on an isokinetic dynamometer (Biodex System 3) pre- and post-exercise ( 1 minute post) to determine isometric strength. Knee extension was performed with the lever arm of the machine fixed at an angle corresponding to 90 degree of knee flexion. The preexercise MVC began with a warm up of three submaximal contractions followed by two maximal contractions. The post MVC ( $\sim 1$ minute post-exercise $)$ involved only two maximal contractions. Each contraction was held for three seconds with 30 seconds rest between each contraction. The MVC value analyzed was the highest MVC torque $(\mathrm{Nm})$ value observed for a respective time point. Participants were familiarized with the MVC testing during their initial screening visit.

\section{Electromyography (EMG)}

Electromyographic (EMG) signals were recorded from the vastus lateralis (VL) of the dominant leg during exercise. A mark was placed on the muscle belly of the VL $66 \%$ of the distance between the anterior-superior iliac crest and the lateral patella. At the site, the skin was shaved, abraded, and cleaned with alcohol wipes. Bipolar electrodes were placed over the muscle belly with an inter-electrode distance of $20 \mathrm{~mm}$. The ground electrode was placed on the $7^{\text {th }}$ cervical vertebrae at the neck. The surface electrodes were connected to an amplifier and digitized (Biopac System, Inc. Goleta, CA, USA). The signal was filtered (low-pass filter $500 \mathrm{~Hz}$; high-pass filter $10 \mathrm{~Hz})$, amplified (1000x) and sampled at a rate of $1 \mathrm{KHz}$. Before the exercise bout, the participant performed two MVCs with the knee extensors at a joint angle of $90^{\circ}$ with $30 \mathrm{~s}$ rest between MVCs on an isokinetic dynamometer (Biodex System 3). The EMG was recorded continuously from the vastus lateralis during each exercise bout. The computer software Labview 7.1 (National Instrument Corporation, Austin, TX) was used to analyze the data. EMG amplitude (root mean square, RMS) and MPF were measured during the first 5 minutes of exercise and the last 5 minutes of exercise and expressed relative to the highest pre-exercise MVC (\%MVC). The first minute and last minute of data collection for each time point were discarded and the 3 minutes between those time points were averaged and used for statistical analyses.

\section{Whole blood lactate (WBL)}

WBL (mmol/L) was indirectly measured using a handheld analyzer (Lactate Plus, Nova Biomedical Corporation, Waltham, MA, USA). Fingertip blood samples were collected before and after ( $\sim 3$ minutes post) exercise as previously described (19).

\section{Hematocrit (HCT)}

Prior to and following the exercise bouts ( $\sim 3$ minutes post), a drop of blood was also drawn up into a capillary tube following the finger sticks for whole blood lactate as previously described (19). 
Muscle thickness (MTH)

B-mode ultrasound measurements of muscle thickness (MTH) were made at 2 anatomical sites on the non-dominant thigh: on the lateral and anterior surface of the thigh at a distance of $50 \%$ between the lateral condyle of the femur and the greater trochanter. Distances between bony landmarks were measured with a tape measure and marked with a pen. All ultrasound measurements were made using a Fukuda Denshi UF-4500 (Tokyo, Japan) ultrasound unit and a $5 \mathrm{MHz}$ linear probe. The probe was coated with transmission gel and placed perpendicular to the tissue interface at the marked sites without depressing the skin. MTH (mm) was determined as the distance from the adipose tissue-muscle interface to musclebone interface. Three measurements of MTH were obtained, printed for analysis, and then averaged. All muscle thickness measurements were taken with the participant standing with their arms and legs relaxed and fully extended (4). Foot placement was held constant by the participant standing in a tracing of two feet, to control for any effect changes in foot position may have on the measurement. In addition, the investigator was blinded to the condition and time point for the analysis of each one of the MTH images.

\section{Thigh circumference (33\%)}

The circumference of the non-dominant thigh was measured with a tape measure at the 33\% site between the top of the patella (knee cap) and the inguinal crease. The $33 \%$ site was measured on the initial visit in the supine position to determine the inflation pressure.

\section{Blood flow restriction}

With the participants in a seated position the blood flow restriction cuffs $(5 \mathrm{~cm}$ wide, KAATSU Master Apparatus.) were applied to the most proximal portion of each thigh at an initial pressure of $50 \mathrm{mmHg}$ (i.e. pressure applied to limb prior to inflation). The cuff was inflated to $50 \mathrm{mmHg}$ for 30 seconds and then deflated for 10 seconds. The cuff was then inflated to $100 \mathrm{mmHg}$ for 30 seconds and then deflated for 10 seconds (unless $100 \mathrm{mmHg}$ was the target pressure). The cycle of cuff inflation/deflation was repeated with the cuff pressure increasing in increments of $40 \mathrm{mmHg}$ until the target inflation pressure was reached. The cuff was inflated to the target inflation pressure prior to exercise and then deflated and removed immediately following exercise. The final pressure was set to a percentage of arterial occlusion estimated from thigh circumference (Table II). To determine estimated arterial occlusion, we used a previous data set $[(18), n=116]$ and plotted thigh circumference with arterial occlusion. This method is likely imperfect but does appear to provide a relative BFR stimulus (22).

Table II. Blood flow restriction pressures

\begin{tabular}{|c|c|c|}
\hline $\begin{array}{c}\text { Thigh circ. } \\
(\mathbf{c m})\end{array}$ & $\begin{array}{c}\text { Pressure used (60\% AO) } \\
(\mathbf{m m H g})\end{array}$ & $\begin{array}{c}\text { Pressure used (40\% AO) } \\
(\mathbf{m m H g})\end{array}$ \\
\hline$<45-50.9$ & 120 & 80 \\
\hline $51-55.9$ & 150 & 100 \\
\hline $56-59.9$ & 180 & 120 \\
\hline$\geq 60$ & 210 & 140 \\
\hline
\end{tabular}

Circ $=$ circumference $; \mathrm{AO}=$ estimated arterial occlusion 
Ratings of perceived exertion (RPE)

A rating of perceived exertion (RPE) was taken at the beginning, middle, and towards the end of exercise using the standard Borg 6-20 scale with methods previously described (23).

\section{Ratings of discomfort}

A rating of discomfort was taken at the beginning, middle, and towards the end of exercise using the Borg Discomfort scale (CR-10+) with methods previously described (23).

\section{$\mathrm{VO}_{2}$ peak testing}

Graded exercise testing (GXT) was conducted using an Excalibur cycle ergometer (Excalibur Sport, Lode, Netherlands) and a metabolic cart (Trueone $2400 \AA$ metabolic measurement system, Parvomedics, Sandy, UT, USA). Before each test, participants had their height measured by a wall mounted stadiometer and body mass measured with an electronic scale (BWB-800, Tanita Corporation of America, Inc., Arlington Heights, IL, USA). Participants wore a polar heart rate belt (Polar T31 Coded ${ }^{\mathrm{TM}}$ Transmitter, Polar Electro, Sacramento, CA, USA) across the chest to monitor their HR throughout. After a one minute warm up, the participant began the GXT. Each stage progressively increased the workload from stage one with a low-intensity of 25 Watts to stage seven with a high-intensity of 325 Watts. The workload was increased by 50 watts following each three-minute stage. This test was conducted twice (separated by approximately a week) to better ensure the accuracy of our workload. The VO2peaks were averaged for presentation in this manuscript.

\section{Statistical analyses}

All data were analyzed using the SPSS 18.0 statistical software package (SPSS Inc., Chicago, IL) with variability represented as standard deviation (SD), unless otherwise stated. A oneway ANOVA was completed for plasma volume, watts, duration, and overall exercise volume to determine if differences existed between conditions.

For MVC, WBL, and MTH a 4 (condition) $\times 2$ (time) repeated measures ANOVA was conducted. A significant result from the repeated measures ANOVA was followed up with a paired sample $t$-test to determine where the difference existed across time within each condition. In addition, a one-way ANOVA determined where differences occurred within each time point across conditions. A 3 (condition) $\times 2$ (time) repeated measures ANOVA was used for EMG. A significant result from the repeated measures ANOVA was followed up with a paired sample $t$-test to determine where the difference existed across time within each condition. In addition, a one-way ANOVA determined where differences occurred within each time point across conditions. A 4 (condition) $\times 3$ (time) repeated measures ANOVA was used for HR. Statistical significance was set at an alpha level of 0.05. All post-hoc comparisons maintained the error rate by Bonferroni correcting the $p$ level.

To compare differences in the perceptual responses (RPE and Discomfort), the Friedman non-parametric test was used to determine if differences existed between conditions at the beginning, middle, or end of exercise. If significant differences existed, Wilcoxon related samples non-parametric tests were used to determine where the difference occurred. Statistical significance for this test was set at an alpha level of 0.05. All post-hoc comparisons maintained the error rate by Bonferroni correcting the $p$ level.

Reliability for MVC, WBL, and MTH was determined from the pre- and post-data from the control visit within each experiment. Those measurements were used in the calculation of the standard error of the measurement (SEM). The minimal difference (MD) needed to be 
considered a real change was calculated from the SEM $(\mathrm{MD}=\mathrm{SEM} \times 1.96 \times \sqrt{2})$. Calculating this with the control condition allowed us to quantify what difference would be needed to ensure that the difference exceeded that which could be expected just through repeated testing (pre/post measurements). Thus, anything exceeding this MD would considered a real change that exceeds the error of the measurement (37).

\section{Results}

\section{Participant characteristics}

Participants $(n=9)$ on average were 25 (4) years old, $178(5) \mathrm{cm}$ tall, $83.2(8) \mathrm{kg}$, a supine measured thigh circumference at the $33 \%$ site of $60.8(4.3) \mathrm{cm}$, and a $\mathrm{VO}_{2}$ peak of $47(7) \mathrm{ml} /$ $\mathrm{kg} / \mathrm{min}$.

\section{Exercise characteristics}

Participants cycled at 202 (36), 97 (26), and 97 (26) watts during the High, 40BFR, and 60BFR conditions. The wattage used during exercise was significantly higher in the High compared to the other conditions ( $p<0.001)$. The duration of exercise was $14(1), 28(3)$, and 30 (0) minutes during the High, 40BFR, and 60BFR conditions. The duration used during exercise was significantly greater in the BFR conditions compared to the High $(p<0.001)$. These differences in wattage and duration allowed the overall exercise volume to be similar between conditions (Table I, $p=0.447$ ). One participant voluntary withdrew from the 40BFR session 18 minutes into exercise. The results were not different when the analyses excluded this participant (data not shown).

\section{Maximal voluntary contraction}

A $4 \times 2$ repeated measures ANOVA found a significant condition $\times$ time interaction with MVC (Fig. 1A, $p=0.046$ ). A one-way ANOVA found no statistical difference between conditions at pre $(p=0.215)$ or post $(p=0.337)$. Paired sample $t$-tests found significant differences from pre-post in all conditions $(p \leq 0.016)$ except for the 60BFR $(p=0.152)$ and the control condition $(p=0.999)$. Although High and 40BFR statistically decreased from baseline, the value did not exceed the error of the measurement (Minimal Difference $=46.2$ $\mathrm{Nm})$.

\section{Muscle thickness}

A $4 \times 2$ repeated measures ANOVA found a significant condition $\times$ time interaction with MTH at the anterior site (Fig. 1B, $p=0.011$ ). A one-way ANOVA found no statistical difference between conditions at pre $(p=0.208)$ or post $(p=0.330)$. Paired sample $t$-tests found significant increases in MTH at the anterior site from pre-post in all conditions $(p \leq$ $0.008)$ except for the 60BFR $(p=0.056)$ and the control condition $(p=0.636)$. All MTH changes at the anterior site in the exercise conditions exceeded the error of the measurement (Minimal Difference $=1 \mathrm{~mm}$ ). A $4 \times 2$ repeated measures ANOVA found a significant condition $\times$ time interaction with $\mathrm{MTH}$ at the lateral site (Fig. $1 \mathrm{C}, p=0.015$ ). A one-way ANOVA found no statistical difference between conditions at pre $(p=0.559)$ or post $(p=$ 0.572 ). Paired sample $t$-tests did not reveal significant differences from pre-post in any condition ( $p \geq 0.064)$, nor did any change at the lateral site exceed the error of the measurement (Minimal Difference $=2 \mathrm{~mm}$ ). 

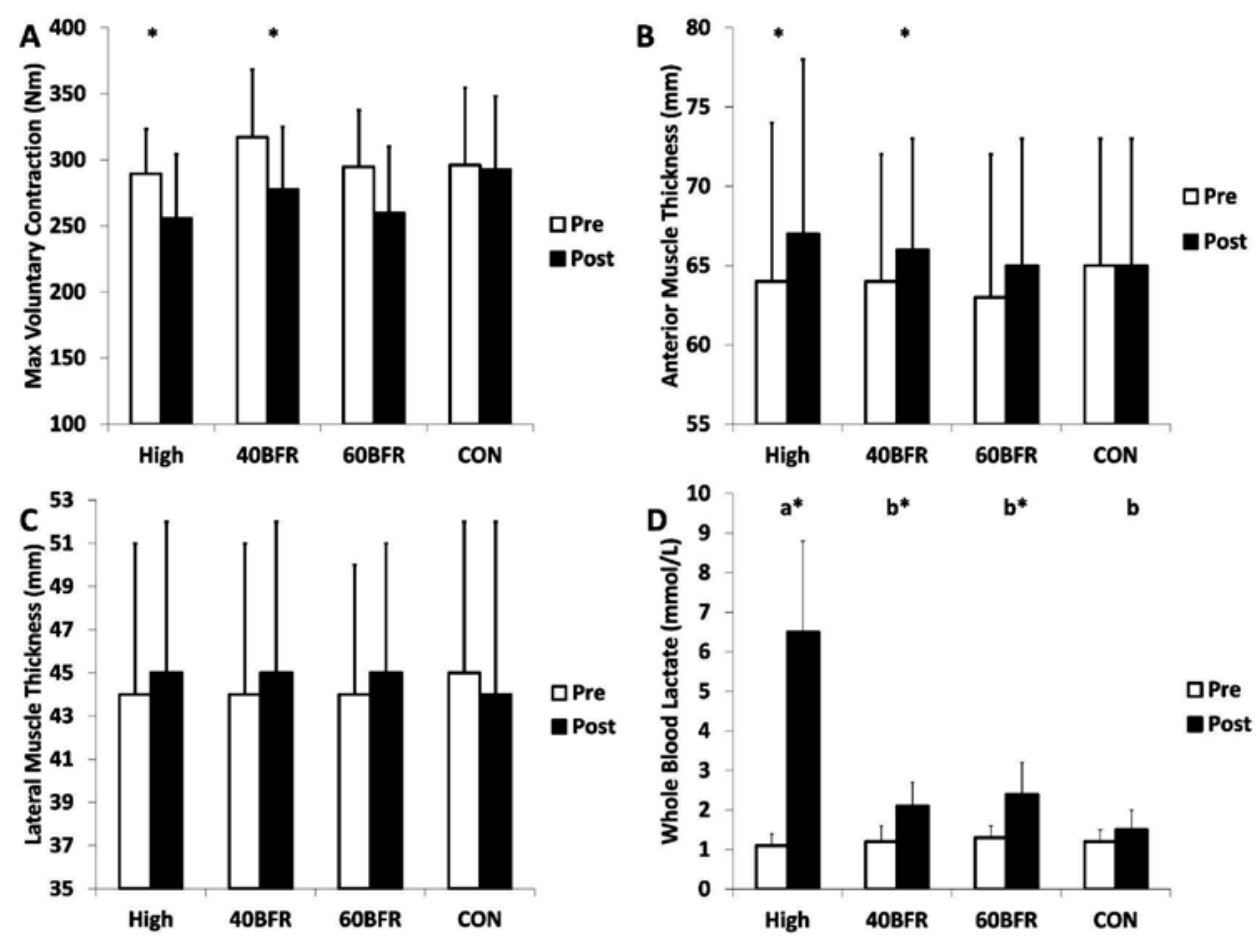

Fig. 1. Mean changes for maximal voluntary contraction (A), anterior thigh muscle thickness (B), lateral thigh muscle thickness (C), and whole blood lactate (D). Conditions with different letters represent significant differences between conditions $(p \leq 0.05)$.*denotes a significant difference from pre to post $(p \leq 0.05)$. Variability represented as standard deviations

\section{Whole blood lactate}

A $4 \times 2$ repeated measures ANOVA found a significant condition $\times$ time interaction with WBL $(p<0.001)$. A one-way ANOVA across the pre-values found no statistical difference between conditions ( $p=0.763$ ). However, a one-way ANOVA did find significant differences across conditions in the post values (Fig. 1D, $p<0.001$ ). Paired sample t-tests found significant increases in WBL from pre-post in all conditions $(p \leq 0.024)$ except for the control condition $(p=0.2)$. All exercise bouts exceeded the minimal difference estimated at 0.8 $\mathrm{mmol} / \mathrm{L}$.

\section{Plasma volume}

A one-way ANOVA found significant differences in plasma volume $(p<0.001)$ across conditions. Pairwise comparisons found that plasma volume was significantly decreased $(p<0.05)-11(5) \%,-12(7) \%$, and $-11(4) \%$ in the High, 40BFR, and 60BFR conditions respectively, compared to control (-2 (2) \%). No other differences were found.

\section{Electromyography (EMG)}

A $3 \times 2$ repeated measures ANOVA found a significant interaction with EMG amplitude (Fig. 2A; $p<0.001$ ). A one-way ANOVA across the EMG amplitudes of the first 5 minutes 
and the last 5 minutes found statistical difference between conditions $(p<0.001)$. Paired sample $t$-tests found a significant increase in EMG amplitude from the first 5 minutes to the last 5 minutes in the High condition $(p=0.003)$, but not the 40BFR $(p=0.999)$ or 60BFR $(p=0.075)$ conditions. A $3 \times 2$ repeated measures ANOVA did not reveal a significant interaction with EMG MPF ( $p=0.129)$. In addition, there was no condition main effect but there was a significant time effect (Fig. 2B, $p<0.001$ ), with MPF increase from the first 5 minutes to the last 5 minutes.
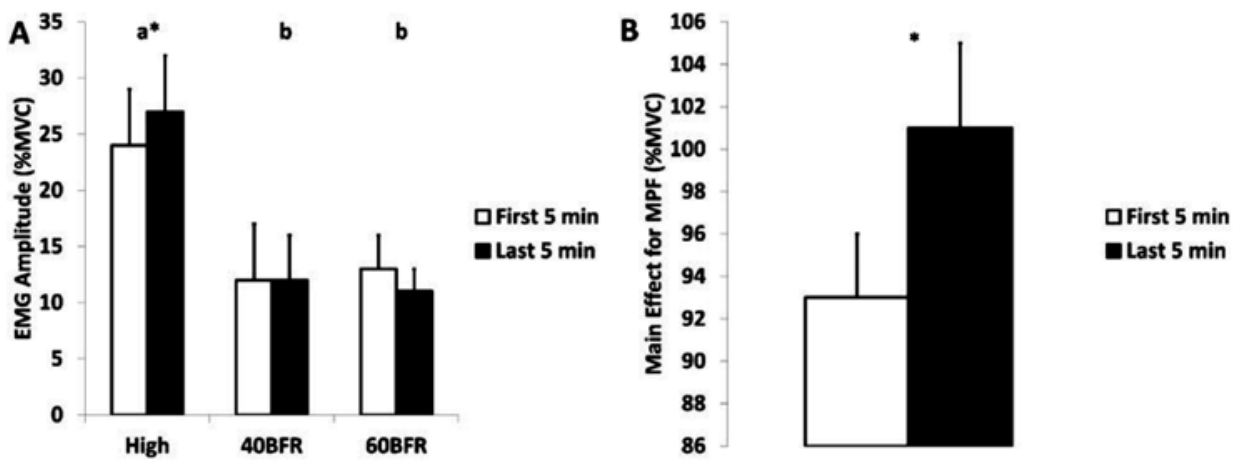

Fig. 2. Mean changes for electromyography amplitude (A) and mean power frequency (B) from the first minutes to the last 5 minutes. Conditions with different letters represent significant differences between conditions $(p \leq 0.05)$. *denotes a significant difference from pre to post $(p \leq 0.05)$.

Variability represented as standard deviations. All values were normalized to the value observed with the pre-exercise maximal voluntary contraction $(\% \mathrm{MVC})$

\section{Heart rate during exercise}

A $3 \times 4$ repeated measures ANOVA found a significant interaction with HR (Table III, $p<$ 0.001). A one-way ANOVA across HR's at every time point found statistical differences between conditions $(p<0.001)$ with High having significantly greater HR's throughout.

Table III. Heart rate response during exercise

\begin{tabular}{|l|c|c|c|c|}
\hline \multicolumn{5}{|c|}{ Heart rate (bpm) $\dagger$} \\
\hline Condition & Beginning & Middle & End & Time \\
\hline High & $143(7)^{\mathrm{a}}$ & $162(10)^{\mathrm{a}}$ & $168(13)^{\mathrm{a}}$ & B vs. M, E; M vs. E \\
\hline $40 \mathrm{BFR}$ & $115(6)^{\mathrm{b}}$ & $131(13)^{\mathrm{b}}$ & $138(13)^{\mathrm{b}}$ & B vs. M, E; M vs. E \\
\hline 60BFR & $111(12)^{\mathrm{b}}$ & $125(17)^{\mathrm{b}}$ & $131(19)^{\mathrm{b}}$ & B vs. M \\
\hline CON & $68(11)^{\mathrm{c}}$ & $67(12)^{\mathrm{c}}$ & $70(14)^{\mathrm{c}}$ & \\
\hline
\end{tabular}

High $=$ high intensity cycling; 40BFR $=$ cycling under $40 \%$ estimated arterial occlusion; 60BFR = cycling under $60 \%$ estimated arterial occlusion pressure; $\mathrm{CON}=$ control. Variability represented as standard deviations.

$\dagger$ denotes a significant condition $\times$ time interaction $(p \leq 0.05)$. Simple effects of condition are noted across each time point. Conditions with different letters represent significant differences between conditions $(p \leq 0.05)$.

Simple effects of time are noted in the far right column in line with each specific condition. The different letters represent significant differences between sets $(p \leq 0.05)$ with $\mathrm{B}=$ beginning ( 3 minutes into exercise),

$\mathrm{M}=$ middle (mid-point of exercise bout), and $\mathrm{E}=$ end (last minute of exercise bout). No letters within each time point across conditions means that the simple effects of condition were not significant following

Bonferroni corrections 


\section{Perceptual responses}

A Friedman non-parametric test found significant differences between conditions in RPE at the beginning $(p=0.004)$, middle $(p=0.005)$, and end of exercise $(p=0.003)$. The High condition recorded the highest RPE throughout (Table IV). A Friedman non-parametric test found no significant differences between conditions for ratings of discomfort (Table IV) at the beginning $(p=0.458)$, middle $(p=0.157)$, or end of exercise $(p=0.356)$.

Table IV. The perceptual response to exercise

\begin{tabular}{|c|c|c|c|}
\hline \multicolumn{4}{|c|}{ Ratings of perceived exertion } \\
\hline Condition & Beginning & Middle & End \\
\hline High & $12-13-14^{\mathrm{a}}$ & $13-15-16^{\mathrm{a}}$ & $14-17-17^{\mathrm{a}}$ \\
\hline 40BFR & $7-9-11^{b}$ & $10-11-13^{b}$ & $11-12-13^{b}$ \\
\hline 60BFR & $7-9-10^{b}$ & $9-12-14^{b}$ & $11-13-15^{b}$ \\
\hline \multicolumn{4}{|c|}{ Ratings of discomfort } \\
\hline Condition & Beginning & Middle & End \\
\hline High & $0.6-2-3$ & $2.2-2.5-5$ & $2.2-5-7$ \\
\hline 40BFR & $0.7-2-5$ & $1.5-3-6.5$ & $2.5-4.1-5$ \\
\hline 60BFR & $1.5-3-5$ & $3.5-6-6.5$ & $3.5-5-8$ \\
\hline
\end{tabular}

High $=$ high intensity cycling; 40BFR $=$ cycling under $40 \%$ estimated arterial occlusion; $60 \mathrm{BFR}=$ cycling under $60 \%$ estimated arterial occlusion pressure. Sets with different letters represent significant differences between conditions $(p \leq 0.05)$. Values represented as the $25^{\text {th }}-50^{\text {th }}-75^{\text {th }}$ percentiles. Beginning ( 3 minutes into exercise); Middle (mid-point of exercise bout), and End (last minute of exercise bout)

\section{Discussion}

When interpreting all of the variables together, the results suggest that high intensity aerobic exercise without BFR provides a greater acute muscular response than work matched low intensity cycling in combination with BFR. Additionally, the heart rate during exercise was higher throughout with high intensity cycling compared to low intensity cycling with BFR, regardless of the pressure applied. Increasing the pressure from $40 \%$ to $60 \%$ of estimated arterial occlusion pressure did not significantly affect heart rate. In addition, it appears that higher (60\% estimated arterial occlusion) relative pressures do not augment any of the muscular responses compared to a lower (40\% estimated arterial occlusion) relative pressure.

\section{Torque}

Several studies have observed large acute decreases in torque following low load resistance exercise in combination with $\operatorname{BFR}(8,22,35,38)$. Furthermore, this large acute decrease immediately post resistance exercise appears to be evidence of fatigue and is thought to provide at least part of the mechanistic rationale for BFR inducing skeletal muscle hypertrophy when it is combined with resistance exercise (25). With respect to low intensity aerobic exercise in combination with BFR, Ogawa et al. (31) observed no decrease in torque following slow or fast walking, suggesting that muscle fatigue may not be a prominent mechanism behind the skeletal muscle hypertrophy observed with low intensity aerobic exercise. 
Our results confirm these findings, in that we found no meaningful (i.e. outside error of measurement) changes in torque regardless of intensity or pressure applied. Given that our measurement was taken $\sim 1$ minute following exercise, it is conceivable that the muscle was able to recover to some extent. Regardless, this is in contrast to the large decreases observed post exercise with resistance training (22).

\section{Muscle swelling}

A number of studies have observed acute increases in markers of muscle swelling following the application of BFR in combination with low load resistance exercise $(9,10,28,41)$. An interesting finding from our laboratory is that the changes in muscle size appear fairly similar across different resistance exercise conditions (unpublished observations). This has also been observed following aerobic exercise in combination with BFR. To illustrate, acute muscle swelling has been observed with slow $(56 \mathrm{~m} / \mathrm{min})$ and fast BFR walking $(87 \mathrm{~m} / \mathrm{min})$ with no differences in muscle swelling between speeds. The results from the current study only found significant meaningful increases in swelling of the anterior thigh for the high intensity and 40 BFR condition, with no differences between conditions. The small sample size likely provides explanation for the lack of significance $(p=0.056)$ for the anterior thigh with 60 BFR. Regardless, the results suggest that increasing the pressure from $40 \%$ to $60 \%$ estimated arterial occlusion has no measurable increase in muscle swelling.

\section{Whole bood lactate}

Several studies using BFR in combination with low load resistance exercise have observed increases in $\operatorname{WBL}(15,26,40)$. The accumulation of this lactate along with other metabolites has been hypothesized to play an important role in skeletal muscle adaptation through increased muscle fiber recruitment (20). With aerobic exercise in combination with BFR, Kumagai et al. (13) previously observed a small increase in lactate levels when cycling at $40 \%$ of $\mathrm{VO} 2_{\max }(\sim 2 \mathrm{mmol} / \mathrm{L}$ increase $)$. The results of the current study also found significant increases in lactate for all conditions, with high intensity cycling producing the greatest increases from baseline. In contrast, the increase observed with cycling under BFR was small and suggests that lactate accumulation is not occurring to a great degree with this type of exercise. This corroborates previous findings observed following slow walking in combination with BFR (24).

\section{Electromyography}

A number of studies have observed increases in EMG amplitude during low load resistance exercise with and without $\operatorname{BFR}(8,9,15,21)$. In contrast to what is observed with resistance training, in the current study EMG amplitude only increased across time with high intensity cycling. With BFR, the activation did not significantly increase from the first 5 minutes to the last 5 minutes, nor did the value ever reach that observed with high intensity cycling. This was observed regardless of the pressure applied and this finding is similar to that observed with resistance training $(8,21)$, in that exercise with BFR produces high levels but rarely reaches the EMG amplitude observed with higher load training (as measured by surface EMG). Higher amplitudes indicate greater motor unit recruitment, firing rate, and/or synchronization throughout the exercise. Given that the increase in EMG amplitude is thought to be due to a metabolic "overload" (i.e. depletion of phosphocreatine stores and decrease in muscle $\mathrm{pH}$ ) within the muscle (33), the current findings suggest that cycling with BFR may not alter the muscular environment enough to augment muscle activation. 
The MPF was increased in all conditions from the beginning to the end of exercise, regardless of the condition. MPF is influenced by many factors, specifically, muscle fiber conduction velocity. Changes in MPF have been indicated as a measure of muscle fiber type recruitment $(14,39)$, although not all reports agree (1). Others have used it as a marker of fatigue. To illustrate, fatigue would cause a decrease in muscle fiber conduction velocity which would ultimately result in a decrease in MPF $(11,34)$. In that sense, the lack of decrease in MPF is in agreement with the lack of measurable change in torque from pre-post exercise. Future research may benefit from visually investigating the power spectrum instead of only evaluating a single quantitative value (7).

\section{Perceptual responses}

The perceptual ratings of exertion were significantly higher with high intensity cycling compared to low intensity BFR cycling, regardless of the pressure applied. In contrast, ratings of discomfort were not significantly different between conditions within each time point. In part, this may be due to variability and the small sample size. Regardless, BFR does not augment the perception of exercise over that observed with high intensity cycling. Further, the pressure applied also had no effect on the perceptual response. Taken together with previous findings (22), BFR may only increase the perceptual response to exercise when compared to a low intensity work matched control. As previously mentioned, one participant prematurely terminated exercise during the 40BFR condition due to extreme discomfort (peak rating of 9). When he returned for the 60BFR condition, he lowered his cadence from 90 to 50 RPM's and was able to successfully complete the exercise bout with minimal discomfort (peak rating of 3). This may seem to suggest that the cadence of exercise is an important variable to account for, at least with respect to the perceptional response to exercise.

\section{Limitations}

In view of the results presented herein, our study does possess some limitations. First, the amount of BFR was estimated for each participant from previous data collected during supine rest but was not directly measured. This was not done due to the complexities involved with measuring changes in blood flow during exercise of the lower body. Regardless, each participant did receive different percentages of arterial occlusion which allowed for the central question of "does applied pressure matter?" to be answered. Second, the lactate levels were estimated from fingertip blood drops. Thus, although this allows us to quantify systemic lactate accumulation, it does not allow us to differentiate differences in lactate accumulation in or around the active muscle tissue. For example, although lactate levels are higher systemically with high intensity cycling, there may be important mechanistic roles for the pooling of lactate around the muscle with BFR during exercise. Lastly, it is noted that these acute load and pressure dependent changes should be investigated further with long-term training studies to determine if acute changes predict or correlate to chronic adaptation.

In conclusion, this experiment suggests that manipulating BFR pressure does not have a large effect on torque, muscle swelling, lactate accumulation, EMG, or the perceptual response to low intensity cycle exercise. Additionally, high intensity cycling substantially increased lactate and muscle activation over time, suggesting that the acute muscular stimulus was greater with high intensity cycling than that observed with work matched low intensity cycling in combination with BFR. The fact that muscle swelling was the only variable that was measurably affected by BFR, supports previous findings that suggests pronounced muscle hypertrophy with BFR occurs only when combined with low load resistance training 
$(16,27)$. Based on our acute muscular responses we wish to suggest that high intensity cycling produces greater muscular stress than that observed with work matched low intensity cycling in combination with BFR. Although speculative until investigated through experiment, these findings seem to suggest that high intensity cycling would likely produce greater muscle adaptation than that observed with work matched low intensity cycling in combination with BFR.

\section{Acknowledgements}

The authors are not aware of any affiliations, memberships, funding, or financial holdings that might be perceived as affecting the objectivity of this manuscript. This study was not supported by any external funding. The authors thank Xin Ye for his help in EMG analysis.

\section{REFERENCES}

1. Aagaard P, Simonsen EB, Andersen JL, Magnusson SP, Halkjaer-Kristensen J, Dyhre-Poulsen P: Neural inhibition during maximal eccentric and concentric quadriceps contraction: effects of resistance training. J. Appl. Physiol. 89, 2249-2257 (2000)

2. Abe T, Fujita S, Nakajima T, Sakamaki M, Ozaki H, Ogasawara R, Sugaya M, Kudo M, Kurano M, Yasuda T, Sato Y, Ohshima H, Mukai C, Ishii N: Effects of low-intensity cycle training with restricted leg blood flow on thigh muscle volume and VO2MAX in young men. J. Sports. Sci. Med. 9, 452-458 (2010)

3. Abe T, Kearns CF, Sato Y: Muscle size and strength are increased following walk training with restricted venous blood flow from the leg muscle, Kaatsu-walk training. J. Appl. Physiol. 100, 1460-1466 (2006)

4. Abe T, Kondo M, Kawakami Y, Fukunaga T: Prediction equations for body composition of japanese adults by b-mode ultrasound. Am. J. of Hum. Biol. 6, 161-170 (1994)

5. Abe T, Sakamaki M, Fujita S, Ozaki H, Sugaya M, Sato Y, Nakajima T: Effects of low-intensity walk training with restricted leg blood flow on muscle strength and aerobic capacity in older adults. J. Geriatr. Phys. Ther. 33, 34-40 (2010)

6. ACSM: American College of Sports Medicine position stand. Progression models in resistance training for healthy adults. Med. Sci. Sports Exerc. 41, 687-708 (2009)

7. Beck TW, Stock MS, Defreitas JM: Shifts in EMG spectral power during fatiguing dynamic contractions. Muscle Nerve 50, 95-102 (2014)

8. Cook SB, Murphy BG, Labarbera KE: Neuromuscular function following a bout of low-load blood flow restricted exercise. Med. Sci. Sports Exerc. 45, 67-74 (2013)

9. Fahs CA, Loenneke JP, Thiebaud RS, Rossow LM, Kim D, Abe T, Beck TW, Feeback DL, Bemben DA, Bemben MG: Muscular adaptations to fatiguing exercise with and without blood flow restriction. Clin. Physiol. Funct. Imaging. 35, 167-176 (2015)

10. Fry CS, Glynn EL, Drummond MJ, Timmerman KL, Fujita S, Abe T, Dhanani S, Volpi E, Rasmussen BB: Blood flow restriction exercise stimulates mTORC1 signaling and muscle protein synthesis in older men. J. Appl. Physiol. 108, 1199-1209 (2010)

11. Komi PV, Tesch P: EMG frequency spectrum, muscle structure, and fatigue during dynamic contractions in man. Eur. J. Appl. Physiol. Occup. Physiol. 42, 41-50 (1979)

12. Konopka AR, Harber MP: Skeletal muscle hypertrophy after aerobic exercise training. Exerc. Sport Sci. Rev. 42, 53-61 (2014)

13. Kumagai K, Kurobe K, Zhong H, Loenneke JP, Thiebaud RS, Ogita F, Abe T: Cardiovascular drift during low intensity exercise with leg blood flow restriction. Acta Physiol. Hung. 99, 392-399 (2012)

14. Kupa EJ, Roy SH, Kandarian SC, De Luca CJ: Effects of muscle fiber type and size on EMG median frequency and conduction velocity. J. Appl. Physiol. 79, 23-32 (1995)

15. Labarbera KE, Murphy BG, Laroche DP, Cook SB: Sex differences in blood flow restricted isotonic knee extensions to fatigue. J. Sports Med. Phys. Fitness 53, 444-452 (2013)

16. Loenneke JP, Abe T, Wilson JM, Thiebaud RS, Fahs CA, Rossow LM, Bemben MG: Blood flow restriction: an evidence based progressive model (Review). Acta Physiol. Hung. 99, 235-250 (2012)

17. Loenneke JP, Fahs CA, Rossow LM, Abe T, Bemben MG: The anabolic benefits of venous blood flow restriction training may be induced by muscle cell swelling. Med. Hypotheses 78, 151-154 (2012) 
18. Loenneke JP, Fahs CA, Rossow LM, Sherk VD, Thiebaud RS, Abe T, Bemben DA, Bemben MG: Effects of cuff width on arterial occlusion: implications for blood flow restricted exercise. Eur. J. Appl. Physiol. 112, 29032912 (2012)

19. Loenneke JP, Fahs CA, Thiebaud RS, Rossow LM, Abe T, Ye X, Kim D, Bemben MG: The acute muscle swelling effects of blood flow restriction. Acta Physiol. Hung. 99, 400-410 (2012)

20. Loenneke JP, Fahs CA, Wilson JM, Bemben MG: Blood flow restriction: the metabolite/volume threshold theory. Med. Hypotheses 77, 748-752 (2011)

21. Loenneke JP, Kim D, Fahs CA, Thiebaud RS, Abe T, Larson RD, Bemben DA, Bemben MG: Effects of exercise with and without different degrees of blood flow restriction on torque and muscle activation. Muscle Nerve 51, 713-721 (2015)

22. Loenneke JP, Thiebaud RS, Fahs CA, Rossow LM, Abe T, Bemben MG: Blood flow restriction does not result in prolonged decrements in torque. Eur. J. Appl. Physiol. 113, 923-931 (2013)

23. Loenneke JP, Thiebaud RS, Fahs CA, Rossow LM, Abe T, Bemben MG: Blood flow restriction: Effects of cuff type on fatigue and perceptual responses to resistance exercise. Acta Physiol. Hung. 101, 158-166 (2014)

24. Loenneke JP, Thrower AD, Balapur A, Barnes JT, Pujol TJ: Blood flow-restricted walking does not result in an accumlation of metabolites. Clin. Physiol. Funct. Imaging. 32, 80-82 (2012)

25. Loenneke JP, Wilson GJ, Wilson JM: A mechanistic approach to blood flow occlusion. Int. J. Sports Med. 31, $1-4(2010)$

26. Loenneke JP, Wilson JM, Balapur A, Thrower AD, Barnes JT, Pujol TJ: Time under tension decreased with blood flow-restricted exercise. Clin. Physiol. Funct. Imaging. 32, 268-273 (2012)

27. Loenneke JP, Wilson JM, Marin PJ, Zourdos MC, Bemben MG: Low intensity blood flow restriction training: a meta-analysis. Eur. J. Appl. Physiol. 112, 1849-1859 (2012)

28. Martin-Hernandez J, Marin PJ, Menendez H, Loenneke JP, Coelho ESMJ, Garcia-Lopez D, Herrero AJ: Changes in muscle architecture induced by low load blood flow restricted training. Acta Physiol. Hung. 100, 411-418 (2013)

29. Mitchell CJ, Churchward-Venne TA, West DW, Burd NA, Breen L, Baker SK, Phillips SM: Resistance exercise load does not determine training-mediated hypertrophic gains in young men. J. Appl. Physiol. 113, 71-77 (2012)

30. Motykie GD, Zebala LP, Caprini JA, Lee CE, Arcelus JI, Reyna J, Cohen EB: A guide to venous thromboembolism risk factor assessment. J. Thromb. Thrombolysis 9, 253-262 (2000)

31. Ogawa M, Loenneke JP, Yasuda T, Fahs CA, Rossow LM, Thiebaud RS, Bemben MG, Abe T: Time course changes in muscle size and fatigue during walking with restricted leg blood flow in young men. JPESM 3, 14-19 (2012)

32. Ozaki H, Kakigi R, Kobayashi H, Loenneke JP, Abe T, Naito H: Effects of walking combined with restricted leg blood flow on mTOR and MAPK signalling in young men. Acta Physiol (Oxf). 211, 97-106 (2014)

33. Suga T, Okita K, Morita N, Yokota T, Hirabayashi K, Horiuchi M, Takada S, Omokawa M, Kinugawa S, Tsutsui $\mathrm{H}$ : Dose effect on intramuscular metabolic stress during low-intensity resistance exercise with blood flow restriction. J. Appl. Physiol. 108, 1563-1567 (2010)

34. Tesch PA, Dudley GA, Duvoisin MR, Hather BM, Harris RT: Force and EMG signal patterns during repeated bouts of concentric or eccentric muscle actions. Acta Physiol. Scand. 138, 263-271 (1990)

35. Thiebaud RS, Yasuda T, Loenneke JP, Abe T: Effects of low-intensity concentric and eccentric exercise combined with blood flow restriction on indices of exercise-induced muscle damage. Interv. Med. Appl. Sci. 5, 53-59 (2013)

36. Van Roie E, Delecluse C, Coudyzer W, Boonen S, Bautmans I: Strength training at high versus low external resistance in older adults: Effects on muscle volume, muscle strength, and force-velocity characteristics. Exp. Gerontol. 48, 1351-1361 (2013)

37. Weir JP: Quantifying test-retest reliability using the intraclass correlation coefficient and the SEM. J. Strength Cond. Res. 19, 231-240 (2005)

38. Wernbom M, Paulsen G, Nilsen TS, Hisdal J, Raastad T: Contractile function and sarcolemmal permeability after acute low-load resistance exercise with blood flow restriction. Eur. J. Appl. Physiol. 112, 2051-2063 (2012)

39. Yamada E, Kusaka T, Tanaka S, Mori S, Norimatsu H, Itoh S: Effects of vascular occlusion on surface electromyography and muscle oxygenation during isometric contraction. J. Sport Rehab. 13, 287-299 (2004)

40. Yasuda T, Abe T, Brechue WF, Iida H, Takano H, Meguro K, Kurano M, Fujita S, Nakajima T: Venous blood gas and metabolite response to low-intensity muscle contractions with external limb compression. Metabolism 59, $1510-1519(2010)$

41. Yasuda T, Loenneke JP, Thiebaud RS, Abe T: Effects of blood flow restricted low-intensity concentric or eccentric training on muscle size and strength. PLoS One 7, e52843 (2012) 